\title{
USING DECISION TREE AND RANDOM FORESTS TO CLASSIFY LAND COVERAGE IN TOMINE RESERVOIR
}

\section{YENNY ESPINOSA GÓMEZ., JAVIER FELIPE MONCADA SÁNCHEZ. \& ORLANDO GARCÍA HURTADO.}

Engineering Faculty, Universidad Distrital Francisco José de Caldas, Bogotá, Colombia

\begin{abstract}
Traditional methods of cover classification should be reviewed and compared against the recent methods proposed for this purpose, so the purpose of this study was to extract thematic information from a Landsat 5 TM satellite image of Tominé Reservoir, and around using two methods of classification and / or regression: Decision Tree (DT) and Random Forests $(R F)$ that were processed and applied to the statistical software R. Levels of thematic accuracy were obtained for each these methods and the comparison was made between them, leading to the conclusion that for the study area, the Random Forests can provide a better extraction of thematic information of land cover which is seen in the values obtained and the results visually, although the classification obtained with Decision Trees is also good.

KEYWORDS: Thematic information extraction, Supervised classification, Decision Tree (DT), Random Forests (RF), Land Cover, Satellite Image, Thematic accuracy
\end{abstract}

Received: Sep 21, 2020; Accepted: Oct 12, 2020; Published: Oct 29, 2020; Paper Id.: IJMPERDOCT20202

\section{INTRODUCTION}

The extraction of thematic information from a satellite image is one of the procedures that make it possible to know the characteristics in relation to the land cover [1] present in a study area in a relatively rapid manner and with a certain degree of reliability if some controls or validations are applied to try to guarantee the best application of one of the extraction methods, taking into account the parameters, aspects, and variables required by the method that is selected and used [2].

Satellite images can be analyzed using different techniques, some of which are traditional, such as conventional methods (parallelepiped method [3], minimum distance method [4], maximum probability method (Mahalanobis distance) [5], the nearest neighbor method [6], among others) and other more recent ones such as non-conventional methods (vector support machines [7], [8], decision trees [9], random forests [10]).

Specifically in this study, two methods are applied to establish the comparison in the results found when carrying out the supervised classification of land cover for the Tominé Reservoir, these are Decision Trees (DT) and Random Forests (RF).

The Decision Tree DT method represents a set of conditions that are organized in a hierarchical way, and which are applied successively from a root to a terminal node or leaf of the tree [11]. Decision trees are represented by a tree-structured network that has been induced from a set of training data. Every decision tree begins with a node to which all the cases in the sample to be classified belong; this node is called the root node. In addition to the root node, internal nodes and terminal nodes can be distinguished, which are also called leaves. The internal nodes correspond to a condition chosen in the set of attributes and each branch of an internal node represents a value of 
the domain of the attribute condition. From the root of the tree to a particular leaf, the path forms a classification rule [12].

The Random Forests RF method was developed and proposed by Leo Breiman and Adele Cutler. This is an ensemble classifier that uses decision trees as base classifiers, each contributing a vote for the assignment of the most frequent class to the input vector; it increases the diversity of the decision trees by growing them from various subsets of data created through the application of a process known as bagging [13].

Bagging (Bootstrap Aggregating) is a technique designed for the creation of training data that randomly resample the original data set with replacement, that is, without removing the selected data set prior to choosing the next subset. Therefore, some data can be used more than once in the training of individual classifiers. Thus RF is less sensitive to slight variations of the input data (changes in training, outliers, noise...), and, at the same time, increases the accuracy of the classifications [12], [14], [15].

When RF creates a classification tree, it uses the best division of a set of random variables or characteristics in the division of each node, since it uses the Gini index, instead of using the best division variables. Thus, this can reduce the accuracy of each individual tree, but it also reduces the correlation between the trees, and therefore the generalization error [14]. In addition, RF-generated trees grow without pruning, which reduces the computational cost [16].

There is no known land cover classification for the study area using the vector support machine method and therefore it has not been possible to establish whether it yields better results than conventional methods. Therefore, it would be important to make a comparison of the two methods mentioned to establish which of the two gives better results in the extraction of thematic land cover information for the Tominé reservoir [17], [18].

The purpose of this study was to extract thematic information from a Landsat 5 TM satellite image of the Tominé reservoir [18], and its surroundings, using these two methods for classification and/or regression: Decision Trees (DT) and Random Forests (RF), by processing the data and techniques in the R [19] statistical software. The initial hypothesis is that when comparing the levels of thematic accuracy obtained by each of the methods, it will be found that the Random Forests method presents better results at those levels than the Decision Tree method and therefore would give a better classification of the data.

\section{DATA AND METHODS}

\section{Study Zone}

The study zone corresponds to the Tominé Reservoir and its surroundings, covering a total area of $900 \mathrm{~km} 2$, this reservoir is an artificial water body that extends through the land of the municipalities of Guatavita and Sesquilé, in the department of Cundinamarca, is located $50 \mathrm{~km}$ northeast of the city of Bogotá, has a length of 18 kilometers long by 4 kilometers wide and 50 meters in average depth, with capacity for $690.000,000$ cubic meters of water, so it has become a reserve of vital importance for the sector; it is also part of the Sabana de Bogotá water system along with the Neusa and Sisga reservoirs. Its capacity is seven times greater than that of the Sisga and Neusa reservoirs, making it the Sabana de Bogotá water reserve with the greatest capacity [17], [20].

The construction of the reservoir began in 1959 and was completed in 1962 when the Empresa de Energía Eléctrica de Bogotá (EEB) began operations. The Tominé Reservoir is the most important project to improve water quality and the use of water resources, with local and regional impact. 
Near the reservoir are several sectors dedicated to crops and there are also the towns of Guatavita, Sesquile [18]. There are five categories of soil cover in the area: Water, Vegetation-Bushes, Crops, Forests, and Pastures. Figure 1 shows the location and delimitation of the study area.

\section{Data used}

The initial data used in this study is a Landsat 5 TM image with Path 8 and Row 57 from March 27, 2002, which has 7002 by 8061 pixels, with a spatial resolution of 28.5 meters by 28.5 meters, a radiometric resolution of 8 bits, and a spectral resolution of seven (7) bands: blue, green, red, near-infrared, mid-infrared, thermal and far-infrared. The reference ellipsoid for the image is the WGS 84 and a Universal Transverse Mercator (UTM) projection referred to as zone 18N.

These bands were cut to obtain a new image of 1052 by 1052 pixels on the study area, covering the zone between the minimum coordinates 523736 North and 612282 East and the maximum coordinates 553736 North and 642282 East, for a total area of $900 \mathrm{~km} 2$. Figure 2 shows the seven bands that make up the image.

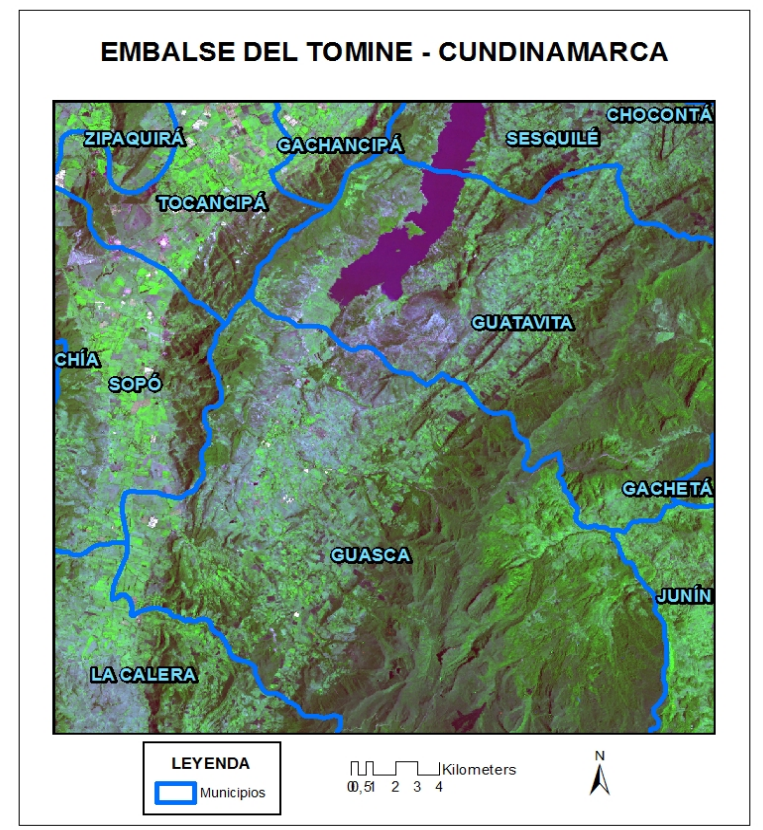

Figure 1. Location and Delimitation of the Study Area. 

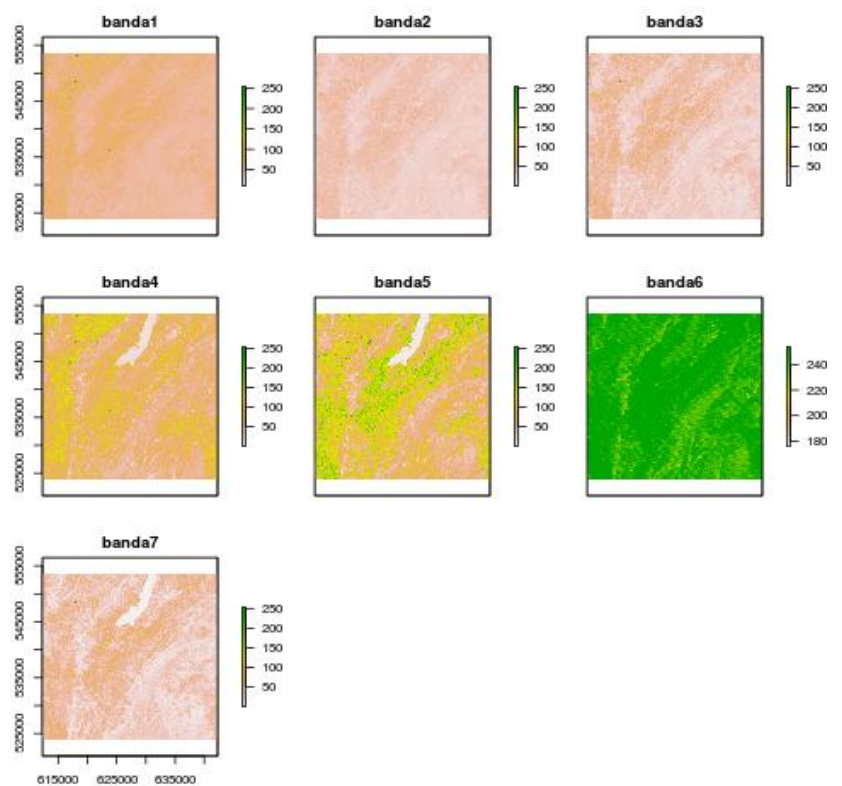

Figure 2: Visualization of the Image Bands.

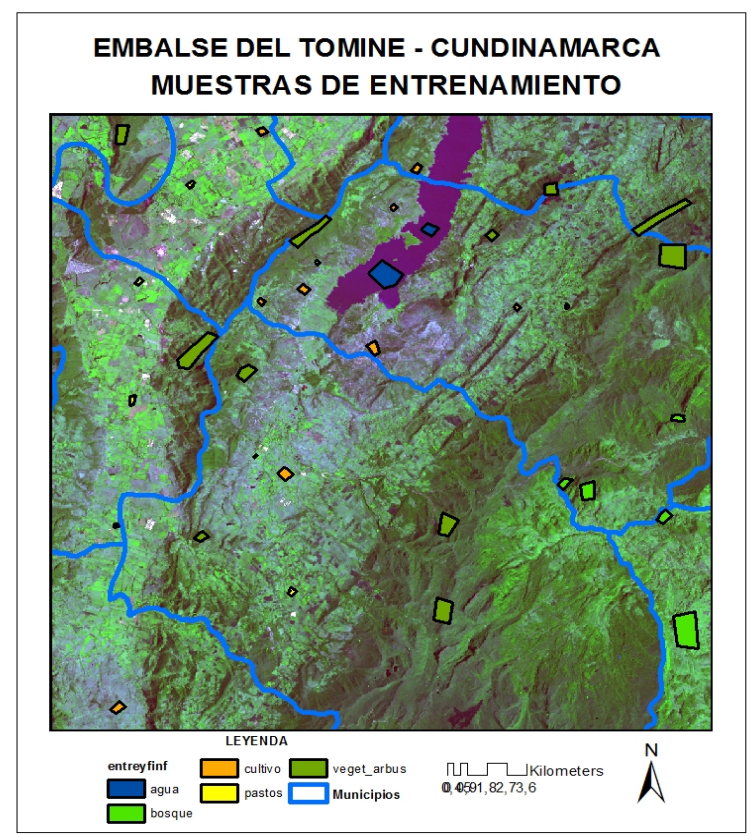

Figure 3. Samples (Polygons) of Training. 


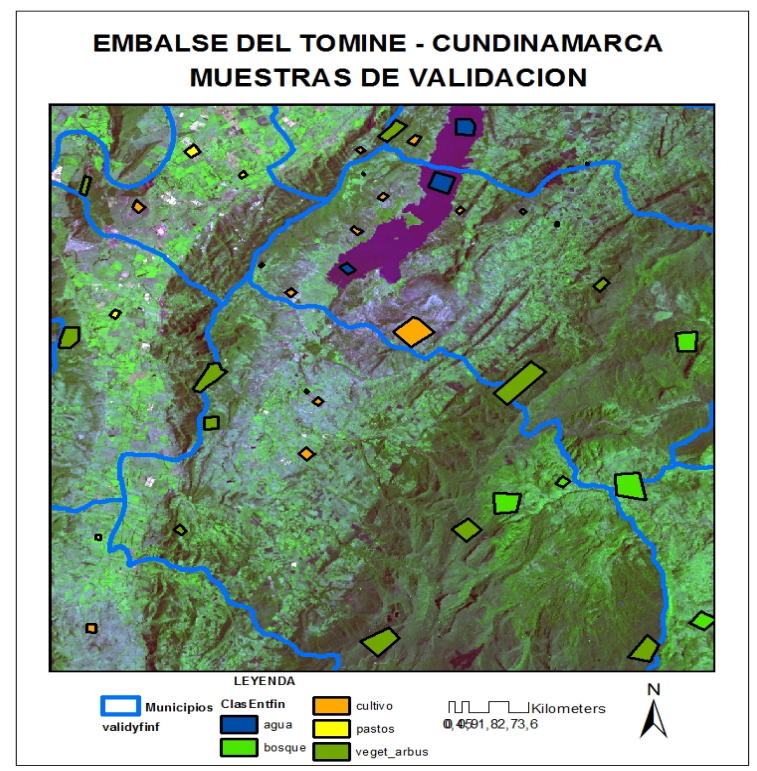

Figure 4: Validation Samples (Polygons)

In addition to this image, two shapefile files (ESRI's spatial data storage structure) were used, which contain the training polygons and the validation polygons, presented in Figures 3 and 4, respectively. These shapefiles were created taking as reference a Land Cover map, from 2008, property of the Instituto Geográfico Agustín Codazzi (IGAC) that was downloaded from the Geographic Information System for National Land Management (SIGOT); and they contain the polygons corresponding to each of the five classes or thematic units of land cover present in the study area, which can be seen in Table 1:

Table 1: Classes of Soil Coverage

\begin{tabular}{|l|c|c|c|}
\hline Clase de Cobertura del Suelo & Índice de la Clase & Áreas de entrenamiento Km2 & Áreas de validación Km2 \\
\hline Agua & 1 & 1,420 & 1,760 \\
\hline vegetación-arbustos & 2 & 6,962 & 8,683 \\
\hline Cultivo & 3 & 1,201 & 2,977 \\
\hline Bosques & 4 & 2,618 & 4,502 \\
\hline Pastos & 5 & 0,424 & 0,542 \\
\hline
\end{tabular}

\section{Methods}

The classification of the land cover for the Tominé Reservoir was carried out and implemented using the statistical program $\mathrm{R}$ which allows the opening, review, and processing of geographical objects in vector and raster structure for the purpose of subsequent analysis in a particular context. The structure of the program is organized in different libraries that must be previously installed in order to be able to execute some processes and techniques required for the object of study; therefore, it was necessary to guarantee the adequate functioning of the libraries: rgdal, sp, raster, rgeos, kernlab, mda, vcd, mass, rpart, e1071, randomForest, foreign, among others.

Figure 5 shows the process flow diagram of the method used to carry out the supervised classification of the land cover using the Decision Tree and Random Forest classification algorithms.

The first process carried out is the "Start of the Supervised Classification", for which the base data (Landsat 5 TM 
image) must be reviewed, the image bands (see Figure 2), the histograms of each image band (see Figure 6), the image statistics and the dispersion between each pair of bands are displayed.

We then moved on to the "Problem definition" in which we defined the target classes to be worked on in this case.

In the processes "Selection of Training Samples" and "Selection of Validation Samples", the most representative training and validation samples of each class were determined from the available shapefile. Then the raster bands of the image and the vector file (shapefile) containing the training samples are loaded for classification.

Afterward, the Separability Analysis between the different classes is performed, to determine if there is any confusion between the separability of the classes

The "Determination of the Efficient Gamma and Cost parameters for the Study sample" was carried out and serves as a basis for the application of the classifiers.

With these data, the "Decision Tree (DT) and Random Forest (RF) Classification" algorithms were executed in the following two processes.

Subsequently, the "Thematic Accuracy Assessment" was carried out for each of the Classifications carried out (by DT and RF), the confusion matrix, the kappa index, and the percentage of elements correctly classified by each of the classifiers were determined in order to compare these values or results for each of the methods used.

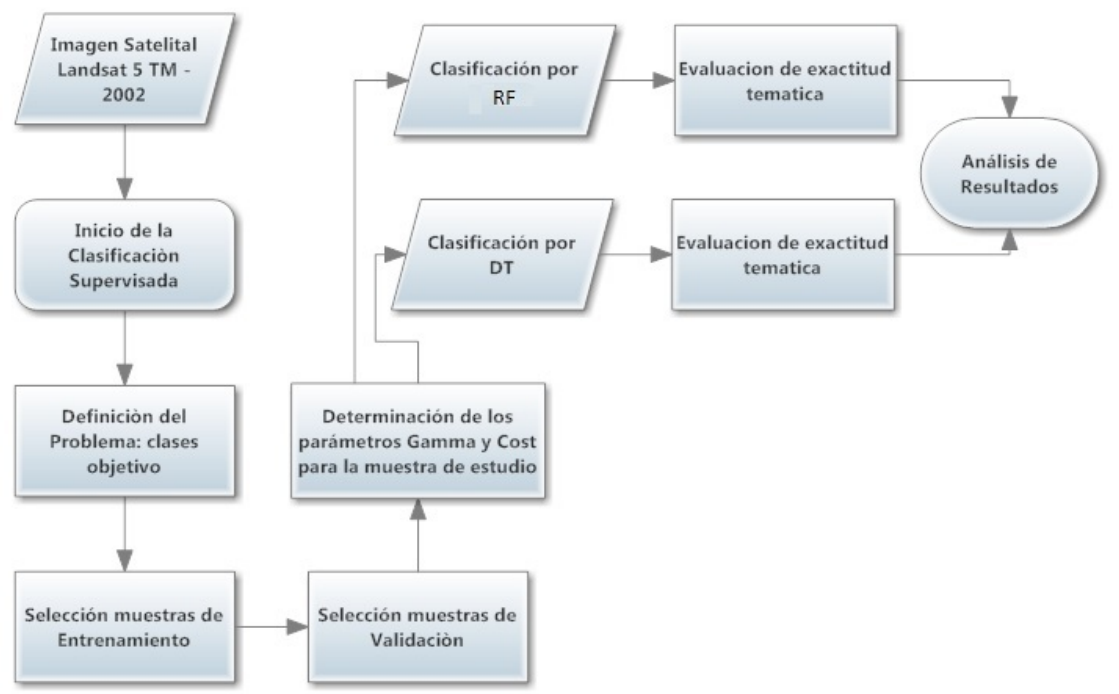

Figure 5. Process Flow Chart used to perform the supervised classification of land cover from DT and RF. 

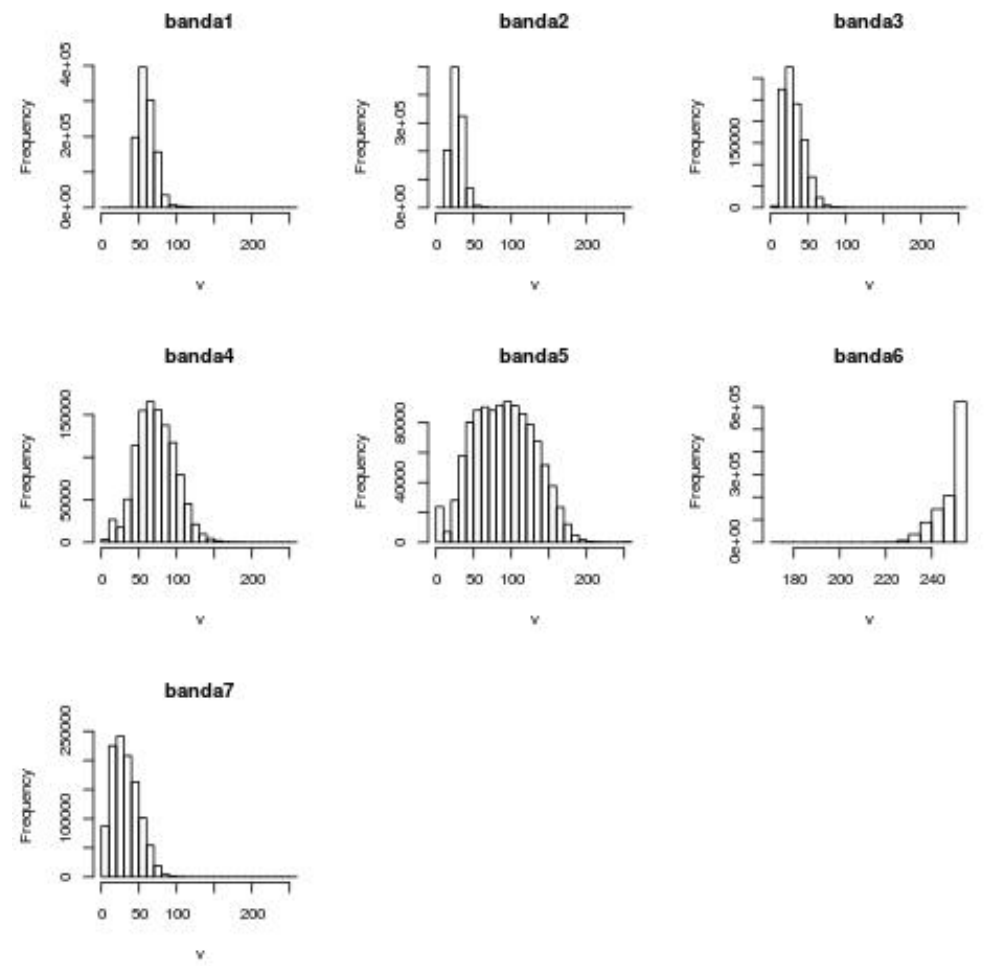

Figure 6. Histograms of each Image Band

Finally, the "Analysis of Results" was carried out, which consists of reviewing the results of the comparison, of the levels of thematic accuracy obtained, in order to establish which of them presents the highest level of thematic accuracy and therefore could be the classification method that offers better results for the case and the area studied..

\section{RESULTS}

The correlation matrix that exists between the bands is shown in Table 2, where it can be seen that there is a high autocorrelation between the bands: Red and Green (0.956), Far Infrared and Medium Infrared (0.949), Blue and Green (0.948), Red and Blue (0.926), and the lowest autocorrelation is presented by the Thermal and Blue bands (0.459). The kernel used was: radial base function and the parameters used were: Cost $(C)=16$ and Gamma $($ sigma $)=1$.

When applying the DT classifier, the confusion matrix is obtained (see Table 3.), in which it can be seen that there is high confusion or mixture or cross between the pixels of the vegetation-bush classes (2) and the forest class (4), medium confusion between the bush vegetation class (2) and crop class (3), and low confusion between bush vegetation class (2) and grass class (5). There is also medium confusion between the crop class (3) and the forest class (4), medium confusion between the crop class (3) and the pasture class (5), and medium confusion between the forest class (4) and the pasture class (5).

Table 2. Correlation matrix between belts.

\begin{tabular}{|l|l|l|l|l|l|l|l|}
\hline Banda & Azul & Verde & Rojo & IRC & IRM & TER & IRL \\
\hline Azul & $\mathbf{1 , 0 0 0}$ & 0,948 & 0,926 & 0,516 & 0,676 & 0,459 & 0,720 \\
\hline
\end{tabular}




\begin{tabular}{|l|l|l|l|l|l|l|l|} 
Verde & 0,948 & $\mathbf{1 , 0 0 0}$ & 0,956 & 0,581 & 0,731 & 0,499 & 0,746 \\
\hline Rojo & 0,926 & 0,956 & $\mathbf{1 , 0 0 0}$ & 0,516 & 0,797 & 0,523 & 0,822 \\
\hline IRC & 0,516 & 0,581 & 0,516 & $\mathbf{1 , 0 0 0}$ & 0,657 & 0,475 & 0,497 \\
\hline IRM & 0,676 & 0,731 & 0,797 & 0,657 & $\mathbf{1 , 0 0 0}$ & 0,655 & 0,949 \\
\hline TER & 0,459 & 0,499 & 0,523 & 0,475 & 0,655 & $\mathbf{1 , 0 0 0}$ & 0,610 \\
\hline IRL & 0,720 & 0,746 & 0,822 & 0,497 & 0,949 & 0,610 & $\mathbf{1 , 0 0 0}$ \\
\hline
\end{tabular}

Thus, when carrying out the classification by DT, the problem classes are: the grass class (5) is the one that presents the worst results in the classification since approximately forty percent of the pixels in this class are mixed or are confused with classes: cultivation (3)

and forest (4); the vegetation-bush class (2) and the forest class (4) have a mix or cross as there is a high number of misclassified pixels; the class Forests (4) also has a considerable number of misclassified pixels in relation to its total pixels. This probably occurred because of some errors in the definition of the training samples.

Table 3. Confusion matrix for the Decision Tree (DT) Classifier

\begin{tabular}{|c|c|c|c|c|c|c|c|c|c|c|}
\hline & \multicolumn{5}{|c|}{ Clase Verdadera } & \multirow{2}{*}{ Total } & \multirow{2}{*}{$\begin{array}{l}\text { Comisió } \\
\text { n }\end{array}$} & \multirow{2}{*}{$\begin{array}{l}\text { Exactitu } \\
\text { d } \\
\text { Usuario }\end{array}$} & \multirow{2}{*}{\begin{tabular}{|l|} 
Riesgo \\
Product \\
or
\end{tabular}} \\
\hline & & 1 & 2 & 3 & 4 & 5 & & & & \\
\hline \multirow{5}{*}{ 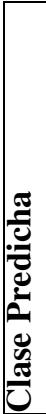 } & 1 & 952 & 1 & 0 & 0 & 0 & 953 & 1 & $99,9 \%$ & $0,1 \%$ \\
\hline & 2 & 0 & 4069 & 82 & 557 & 0 & 4708 & 639 & $86,4 \%$ & $13,6 \%$ \\
\hline & 3 & 0 & 225 & 1218 & 78 & 17 & 1538 & 320 & $79,2 \%$ & $20,8 \%$ \\
\hline & 4 & 0 & 560 & 63 & 1718 & 52 & 2393 & 675 & $71,8 \%$ & $28,2 \%$ \\
\hline & 5 & 0 & 11 & 56 & 119 & 109 & 295 & 186 & $36,9 \%$ & $63,1 \%$ \\
\hline \multicolumn{2}{|c|}{ Total } & 952 & 4866 & 1419 & 2472 & 178 & 9887 & & & \\
\hline \multicolumn{2}{|c|}{ Omisión } & 0 & 797 & 201 & 754 & 69 & & 1821 & & \\
\hline \multicolumn{2}{|c|}{$\begin{array}{l}\text { Exactitud } \\
\text { Productor }\end{array}$} & $\begin{array}{l}100,0 \\
\%\end{array}$ & $83,6 \%$ & $85,8 \%$ & $69,5 \%$ & $61,2 \%$ & & & & \\
\hline \multicolumn{2}{|c|}{$\begin{array}{l}\text { Riesgo } \\
\text { Usuario }\end{array}$} & $0,0 \%$ & $16,4 \%$ & $14,2 \%$ & $30,5 \%$ & $38,8 \%$ & & & & \\
\hline
\end{tabular}


Table 4: Confusion matrix for the Random Forests (RF) Classifier

\begin{tabular}{|c|c|c|c|c|c|c|c|c|c|c|}
\hline & \multicolumn{5}{|c|}{ Clase Verdadera } & \multirow[b]{2}{*}{ Total } & \multirow{2}{*}{$\begin{array}{c}\text { Comisió } \\
\mathbf{n}\end{array}$} & \multirow{2}{*}{$\begin{array}{c}\text { Exactitu } \\
\text { d } \\
\text { Usuario }\end{array}$} & \multirow{2}{*}{$\begin{array}{c}\text { Riesgo } \\
\text { Product } \\
\text { or }\end{array}$} \\
\hline & & 1 & 2 & 3 & 4 & 5 & & & & \\
\hline \multirow{5}{*}{ 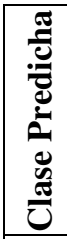 } & 1 & 993 & 0 & 0 & 0 & 0 & 993 & 0 & $100,0 \%$ & $0,0 \%$ \\
\hline & 2 & 0 & 3978 & 63 & 722 & 17 & 4780 & 802 & $83,2 \%$ & $16,8 \%$ \\
\hline & 3 & 0 & 248 & 1259 & 41 & 29 & 1577 & 318 & $79,8 \%$ & $20,2 \%$ \\
\hline & 4 & 0 & 422 & 77 & 1720 & 31 & 2250 & 530 & $76,4 \%$ & $23,6 \%$ \\
\hline & 5 & 0 & 18 & 45 & 33 & 191 & 287 & 96 & $66,6 \%$ & $33,4 \%$ \\
\hline \multicolumn{2}{|c|}{ Total } & 993 & 4666 & 1444 & 2516 & 268 & 9887 & & & \\
\hline \multicolumn{2}{|c|}{ Omisión } & 0 & 688 & 185 & 796 & 77 & & 1746 & & \\
\hline \multicolumn{2}{|c|}{$\begin{array}{l}\text { Exactitud } \\
\text { Productor }\end{array}$} & $\begin{array}{c}100,0 \\
\%\end{array}$ & $85,3 \%$ & $87,2 \%$ & $68,4 \%$ & $71,3 \%$ & & & & \\
\hline \multicolumn{2}{|c|}{$\begin{array}{c}\text { Riesgo } \\
\text { Usuario }\end{array}$} & $0,0 \%$ & $14,7 \%$ & $12,8 \%$ & $31,6 \%$ & $28,7 \%$ & & & & \\
\hline
\end{tabular}

One class that presents a good classification in the DT confusion matrix is the Water class (1) since it presents a user accuracy of $99.9 \%$ and a producer accuracy of $100 \%$, therefore it has a high level of thematic accuracy.

\section{Clases obtenidas mediante arboles de decision}

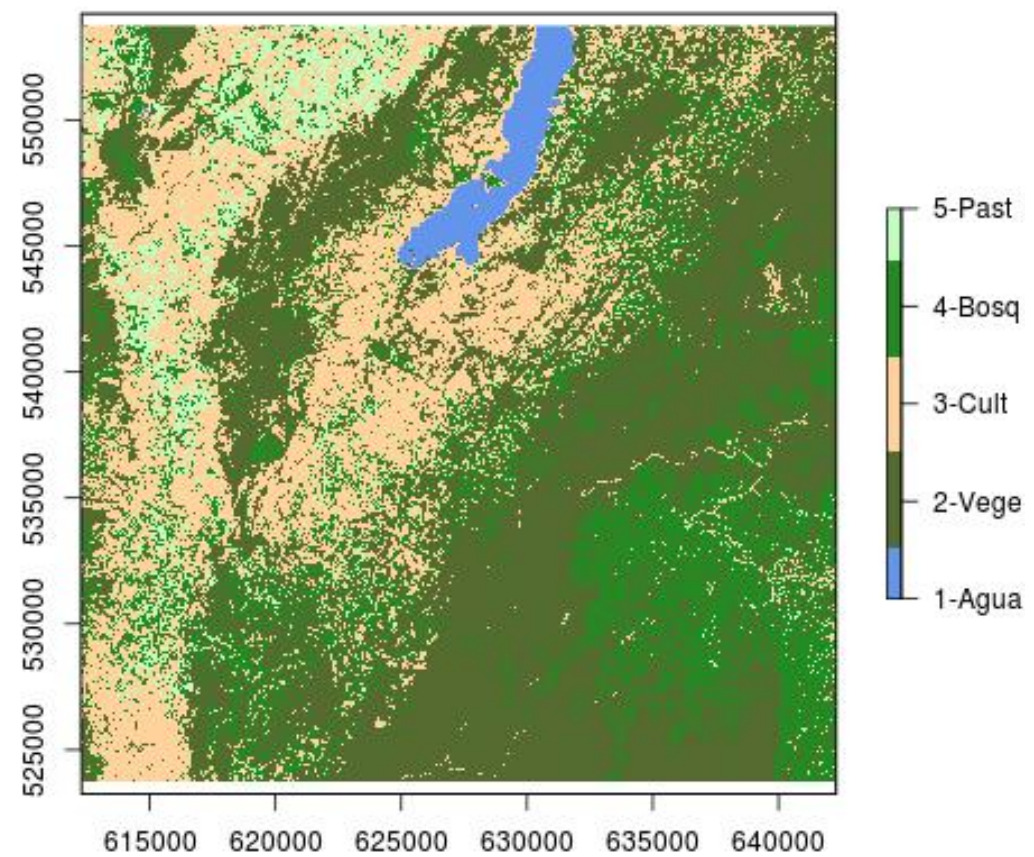

Figure 7: Classification of Coverage obtained through Decision Trees (DT) 
Clases obtenidas mediante Random Forest

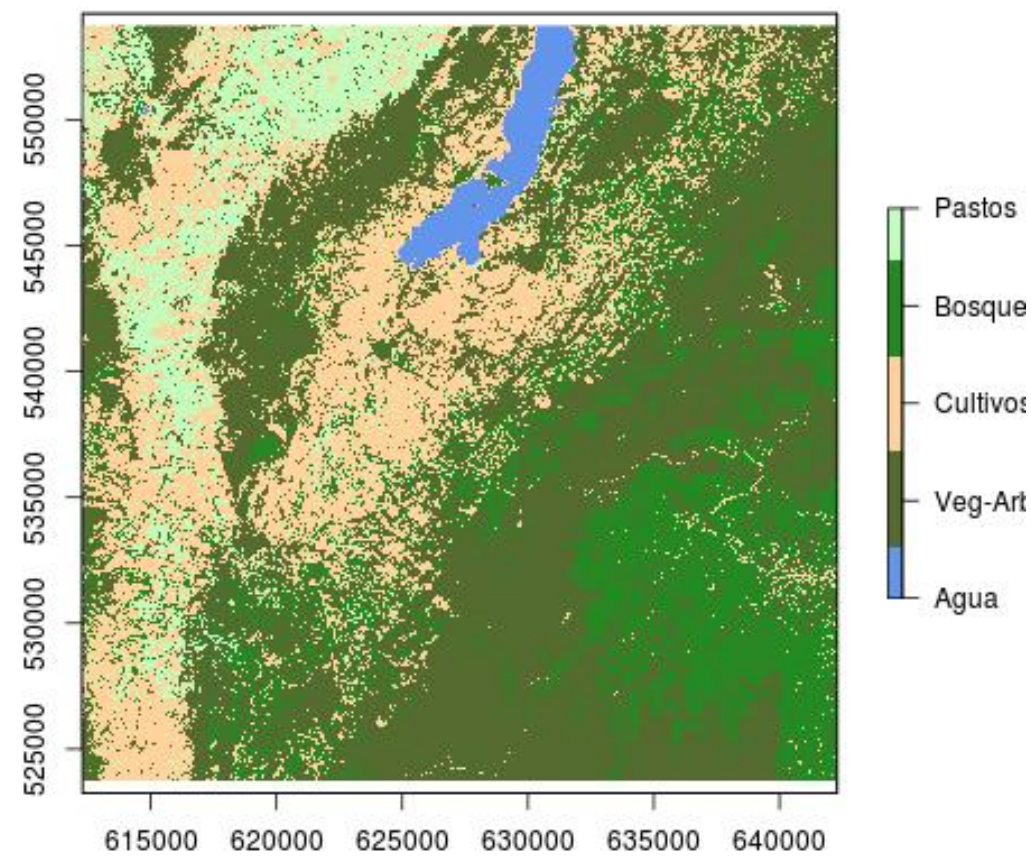

Figure 8: Coverage classification obtained through Random Forests (RF)

randfor
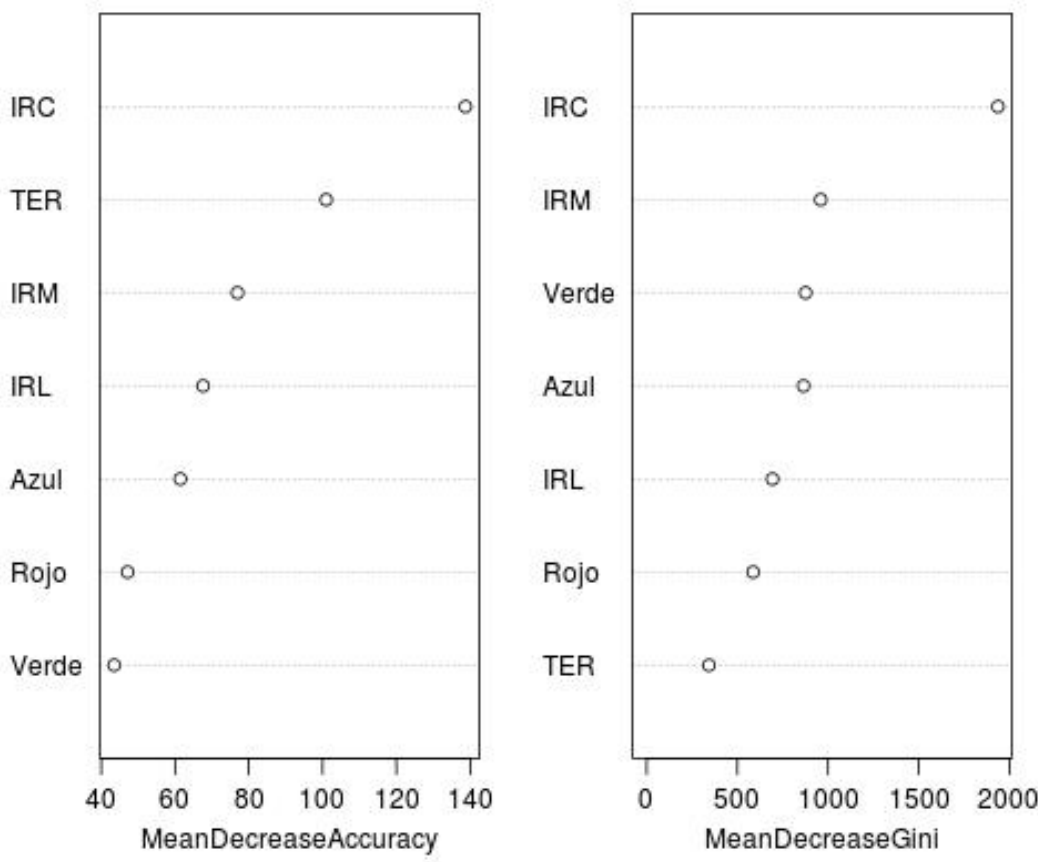

Figure 9: The model was obtained for the Classification of Land Cover by Random Forests.

Also when applying the DT classifier, the correctly classified percentage $(\mathrm{PCC}=80.66)$ and the kappa value $(\mathrm{K}=$ 
0.7263211) were obtained, which indicates that the reliability obtained is good.

When applying the RF classifier, the confusion matrix is obtained (see Table 4.), in which it can be seen that there is high confusion or mixing or crossing between the pixels of the vegetation-bush classes (2) and the forest class (4), medium confusion between the vegetation-bush classes (2) and the crop class (3), and low confusion between the vegetation-bush classes (2) and the pasture class (5). There is also low confusion between the crop class (3) and the forest class (4), low confusion between the crop class (3) and the pasture class (5), and medium confusion between the forest class (4) and the pasture class (5).

Thus, when carrying out the RF classification, the problem classes are the grass class (5) is the one that presents the worst results in the classification, since approximately one-third of the pixels in this class are mixed or confused with the other classes (vegetation-bush (2), crop (3) and forest (4) except for water (1)); the vegetation-bush class (2) and forest (4) present a mixture of crossover since there is a high number of misclassified pixels; the forest class (4) also presents a considerable number of misclassified pixels in relation to the total number of its pixels. This probably occurred because of some errors in the definition of the training samples.

A class that presents excellent classification in the RF confusion matrix, is the Water class (1) since it presents a user accuracy of $100 \%$ and a producer accuracy of $100 \%$, therefore it has a very high level of thematic accuracy.

Also when applying the RF classifier, the correctly classified percentage $(\mathrm{PCC}=81.30)$ and the kappa value $(\mathrm{K}=$ 0.7396853 ) were obtained, which indicates that the reliability obtained is good and is a little better than that obtained by the DT classifier. In addition, the RF algorithm classified 75 more pixels in relation to the number of pixels classified by DT.

Table 5: Thematic accuracy assessment rates.

\begin{tabular}{|l|l|l|}
\hline Índice & Clasificador DT & Clasificador RF \\
\hline PCC & 80.66 & 81.30 \\
\hline KAPPA & 0.7263211 & 0.7396853 \\
\hline
\end{tabular}

When applying the two classifiers, it can be seen (see Table 5.) that the percentage correctly classified and the kappa index are slightly higher for the RF algorithm in relation to the DT algorithm. When comparing the confusion matrices obtained for each one of the classifiers, it can be seen, in general, that there is a small increase in the total number of correctly classified pixels when applying the RF algorithm, although in RF there is a small (minimum) reduction in the user's accuracy for the vegetation-bush class (2) in relation to DT and also in RF there is a small (minimum) reduction in the producer's accuracy for the forest class (4) in relation to DT.

Figures 7 and 8 show the land cover classifications obtained by the Decision Tree (DT) and Random Forests (RF) classifiers, respectively. Visually, it can be seen that the result of the RF classification is presumably much better than the result of the DT classification since there is less mixing or visual confusion of the pixels; although the result of the two land cover classifications is very similar and gives very similar values.

Figure 9 shows the model obtained for the Land Cover Classification, applying the Random Forests (RF) classifier. In this model, it can be seen that the near-infrared and thermal bands are the ones that contribute most to the classification. 


\section{DISCUSSIONS}

It can be seen that the result in figures obtained from the land cover classification for each of the methods applied is similar and does not differ much from each other, that is, although the RF classifier presents a higher value in the thematic accuracy (PCC) and in the Kappa index, these do not show a great or high variation compared to those obtained when the DT classifier is used, therefore, in this case, one or the other of the two classifications could be taken if the work does not require high degrees of accuracy.

In reviewing the result of the two classifications against the 2008 Land Cover map, which was downloaded from the Geographic Information System for National Land Management (SIGOT); several differences are seen mainly in the grass class which is clearly delimited in the coverage map while the classification obtained by DT is mixed with the other classes except for water, this could be a consequence of the difference in the date of the data since the coverage map is from the year 2008 while the Landsat 5 TM image used is from the year 2002, In addition, the soil cover can have rapid variation and/or change in this zone for these two periods of time due to agricultural activities (the presence of crops and pastures) and in general, anthropic factors that occur in the Bogotá savannah for social and economic reasons. In the classification obtained by RF, the grass class is no longer so mixed up with the other classes and visually it looks better.

Although when applying the Random Forest (RF) classifier, overall thematic accuracy is obtained a little higher than the Decision Tree (DT) class, the grass class (5) continues to be the one with the worst classification since it presents confusion with the vegetation-bush (2), crop (3) and forest (4) classes, therefore RF still does not solve this problem. This confusion could be explained by the fact that it seems that few training and validation samples were taken for the forest class, and that improving these samples could improve the classification [13], and that the reflectance between these classes could be taken as similar by the software when performing the process.

The high confusion between the pixels of the Shrub Vegetation class (2) and the forest class (4) could be explained by the fact that the reflectance levels are similar and the separation between classes requires more definition elements and probably more training and validation samples, in addition to the fact that it is not easy to perceive the differentiation between these classes without previous work or field visits to verify the differences between them and therefore the exact positions of them in the study area or to see the possibility of integrating these classes into one.

Something similar occurs between the crop classes and the forest and pasture classes, which is medium confusion, probably because the training and validation samples were not separated enough or not enough to allow a better classification.

The image classified by the Decision Tree (DT) method does not have well-defined boundaries of the different land cover classes in some areas, while the image classified by the Random Forest (RF) method does have better delimitation or definition of the different classes. However, it is evident that it is necessary to have fieldwork prior to classification to better determine the absolute location of the training and validation samples, in addition to having other sources of information and not only the information that is in the satellite image, because this is not enough to perform a good digital classification process of images.

Another interesting situation is to go to the field after the application of the classification to validate the results of the coverage classification by the two methods used in order to check which of them is really giving better results and specifically which classes or types of coverage of the Tominé Reservoir are better classified than others and which are the 
problems and why.

\section{CONCLUSSIONS}

In the case of study, the methods used here of supervised classification not conventional: Decision Trees (DT) and Random Forests (RF) give very similar results both in the numerical controls (percentage correctly classified, kappa index) and visually for this process of classification of the ground cover for the Tomine reservoir; although if one is strict in the revision of the values obtained in the global thematic accuracy it is appreciated that the Random Forests have better results, therefore the initial hypothesis is checked.

The adequate and correct determination of the training and validation samples is a condition for obtaining better results since you are participating as a fundamental actor in the application of these classifiers and guarantee to have better results.

It is crucial to have good reference data on the current or recent state of the variable studied (in this case land cover) to have greater certainty in the definition of classes, the training sample, of the validation points, and in general to have a slightly wider knowledge of the studied phenomenon in order to make better decisions in the application of the different classification methods and in turn to better analyze the results found and to be able to determine more accurately whether or not the classifiers used in determining the land cover of the study area (Tominé Reservoir) are valid. It is also key to have the possibility of having recent information from a previous field visit to the area studied and any other additional information that contributes to a better knowledge of the study area and therefore to the more accurate definition or the delimitation of the land cover classes.

The free software $\mathrm{R}$ is increasingly a good support tool for spatial data processing (both raster and vector) when there is a low budget in the development of projects or as in this case for an academic and research exercise. In addition to many scripts that are available on the network and free access that allow applying processes that improve the classification of satellite images and the extraction of information from them.

\section{REFERENCES}

1. H. Demirel and G. Anbarjafari, "Satellite image resolution enhancement using complex wavelet transform," IEEE Geosci. Remote Sens. Lett., vol. 7, no. 1, pp. 123-126, 2009.

2. S. Abburu and S. B. Golla, "Satellite image classification methods and techniques: A review," Int. J. Comput. Appl., vol. 119, no. $8,2015$.

3. M. Xiang, C.-C. Hung, M. Pham, B.-C. Kuo, and T. Coleman, "A parallelepiped multispectral image classifier using genetic algorithms,” in Proceedings. 2005 IEEE International Geoscience and Remote Sensing Symposium, 2005. IGARSS'05., 2005, vol. 1, pp. 4--pp.

4. F. S. Al-Ahmadi, A. S. Hames, and others, "Comparison of four classification methods to extract land use and land cover from raw satellite images for some remote arid areas, Kingdom of Saudi Arabia,” Earth, vol. 20, no. 1, pp. 167-191, 2009.

5. A. Ahmed, M. Muaz, M. Ali, M. Yasir, S. Ullah, and S. Khan, "Mahalanobis distance and maximum likelihood based classification for identifying tobacco in Pakistan," in 2015 7th International Conference on Recent Advances in Space Technologies (RAST), 2015, pp. 255-260.

6. T. Hastie and R. Tibshirani, "Discriminant adaptive nearest neighbor classification and regression," in Advances in Neural Information Processing Systems, 1996, pp. 409-415. 
7. J. R. Otukei and T. Blaschke, "Land cover change assessment using decision trees, support vector machines and maximum likelihood classification algorithms," Int. J. Appl. Earth Obs. Geoinf., vol. 12, pp. S27--S31, 2010.

8. S. M. Moorthi, I. Misra, R. Kaur, N. P. Darji, and R. Ramakrishnan, "Kernel based learning approach for satellite image classification using support vector machine," in 2011 IEEE Recent Advances in Intelligent Computational Systems, 2011, pp. $107-110$

9. S. E. Sesnie, P. E. Gessler, B. Finegan, and S. Thessler, "Integrating Landsat TM and SRTM-DEM derived variables with decision trees for habitat classification and change detection in complex neotropical environments," Remote Sens. Environ., vol. 112, no. 5, pp. 2145-2159, 2008.

10. N. Horning and others, "Random Forests: An algorithm for image classification and generation of continuous fields data sets, " in Proceedings of the International Conference on Geoinformatics for Spatial Infrastructure Development in Earth and Allied Sciences, Osaka, Japan, 2010, vol. 911.

11. C.-C. Yang et al., "Application of decision tree technology for image classification using remote sensing data," Agric. Syst., vol. 76, no. 3, pp. 1101-1117, 2003.

12. V. RODRÍGUEZ GALIANO and M. CHICA RIVAS, "Clasificación de imágenes de satélite mediante software libre: nuevas tendencias en algoritmos de Inteligencia Artificial," in XV Congreso Nacional de Tecnologías de la Información Geográfica, Madrid, AGE-CSIC, 2012, pp. 19-21.

13. G. Liang, X. Zhu, and C. Zhang, “An empirical study of bagging predictors for different learning algorithms,” in Proceedings of the Twenty-Fifth AAAI Conference on Artificial Intelligence, 2011, pp. 1802-1803.

14. L. Breiman, "Random forests," Mach. Learn., vol. 45, no. 1, pp. 5-32, 2001.

15. V. F. Rodriguez-Galiano, B. Ghimire, J. Rogan, M. Chica-Olmo, and J. P. Rigol-Sanchez, "An assessment of the effectiveness of a random forest classifier for land-cover classification," ISPRS J. Photogramm. Remote Sens., vol. 67, pp. 93-104, 2012.

16. V. F. Rodríiguez Galiano and Others, Metodología basada en teledetección, SIG y geoestadística para cartografía y análisis de cambios en las cubiertas del suelo de la provincia de Granada. Granada: Universidad de Granada, 2012.

17. M. Rodríguez, J. Brisson, G. Rueda, and M. S. Rodríguez, "Water quality improvement of a reservoir invaded by an exotic macrophyte,” Invasive Plant Sci. Manag., vol. 5, no. 2, pp. 290-299, 2012.

18. G. E. Bogotá, "Embalse de Tominé," 2020. [Online]. Available: https://www.grupoenergiabogota.com/eeb/index.php/sostenibilidad/gestion-sostenible/embalse-de-tomine.

19. R Project, “R Project,” 2020. [Online]. Available: https://www.r-project.org/.

20. S. E. Vargas Cubillos, "Análisis espacial por medio de tecnología SIG en la zona sur del embalse de Tominé: Municipio de Guatavita," 2018. 